\title{
desenvolvimento $\widehat{\mathrm{em}}$ QUESTAOO
}

\section{Áreas Naturais Particulares em Ambientes Urbanos: Uma Revisão Bibliográfica}

http://dx.doi.org/10.21527/2237-6453.2019.46.287-298

Recebido em: 18/11/2017

Aceito em: $17 / 8 / 2018$

\author{
Niklas Werner Weins, ${ }^{1}$ Ricardo Gomes Luiz, ${ }^{2}$ Leticia Costa de Oliveira Santos, ${ }^{3}$ \\ Judith Karina Agudelo Fernandez, ${ }^{4}$ Eloy Fassi Casagrande Junior, ${ }^{5}$ \\ Maclovia Correa da Silva, ${ }^{6}$ Silvestre Labiak Junior, ${ }^{7}$ Christian Luiz da Silva ${ }^{8}$
}

\begin{abstract}
RESUMO
As áreas naturais urbanas proveem benefícios significativos para o bem-estar humano. Quando a importância dessas áreas é em grande parte reconhecida pelo conhecimento científico, incentivos legais como as Reservas Particulares de Patrimônio Natural Municipal (RPPNM) no município de Curitiba, PR são uma prova prática de uma tendência de medidas para conservar estes valores. O presente artigo é uma revisão bibliográfica que explora a documentação de seis temas relacionados a estes benefícios considerados emergentes nas políticas públicas urbanas. Os resultados desta análise expressam o reconhecimento geral dos benefícios para o bem-estar humano mediante índices, mas também deixam clara uma necessidade da consideração dos benefícios no planejamento de cidades resilientes e mais biodiversas.
\end{abstract}

Palavras-chave: Áreas naturais. Ambientes urbanos. Serviços ecossistêmicos.

\section{PRIVATE NATURAL AREAS IN URBAN ENVIRONMENTS: A LITERATURE REVIEW}

\begin{abstract}
Urban natural areas provide significant benefits to human well-being. While the importance of these areas is largely acknowledged by scientific knowledge, legal incentives such as the Private Reserves of Municipal Natural Heritage (RPPNM) in the city of Curitiba, Brazil are a practical proof of a trend of measures to conserve these values. This bibliographical review explores the documentation of six topics related to these benefits considered as emerging in urban public policies. The results of the analysis point to the general recognition of the benefits to human well-being through indexes, but also points out the need to consider the benefits of planning resilient and more biodiverse cities.
\end{abstract}

Keywords: Natural areas. Urban environments. Ecosystem services.

\footnotetext{
${ }^{1}$ Bachelor of Arts in Economics and Politics da Ruhr University, Bochum, Alemanha. Aluno do Programa de Pós-Graduação em Tecnologia e Sociedade (Mestrado) da Universidade Tecnológica Federal do Paraná (UTFPR).weinsniklas@gmail.com

${ }^{2}$ Mestre e aluno de Doutorado pelo Programa de Pós-Graduação em Tecnologia e Sociedade da Universidade Tecnológica Federal do Paraná (UTFPR).RicardoGomesLuiz@gmail.com

${ }^{3}$ Bacharel em Composição Paisagística pela Universidade Federal do Rio de Janeiro e em Arquitetura e Urbanismo pela Universidade Tecnológica Federal do Paraná. Aluna do Programa de Pós-Graduação em Tecnologia e Sociedade (mestrado) da Universidade Tecnológica Federal do Paraná (UTFPR). Icos.leticia@gmail.com

${ }^{4}$ Ingeniera Industrial pela Universidad Nacional Experimental del Tachira, San Cristóbal, Venezuela. Aluna do Programa de Pós-Graduação em Tecnologia e Sociedade (Mestrado) da Universidade Tecnológica Federal do Paraná (UTFPR). agudelokarinassl@gmail.com

${ }^{5}$ Doutor em Engenharia de Recursos Minerais e Meio Ambiente pela Universidade de Nottingham, Grã-Bretanha. Professor do Programa de Pós-Graduação em Tecnologia e Sociedade da Universidade Tecnológica Federal do Paraná (UTFPR). eloy.casagrande@gmail.com

${ }^{6}$ Doutora em Arquitetura e Urbanismo pela Universidade de São Paulo (USP. Professora do Programa de Pós-Graduação em Tecnologia e Sociedade da Universidade Tecnológica Federal do Paraná (UTFPR). macarini@utfpr.edu.br

7 Doutor em Engenharia e Gestão do Conhecimento pela Universidade Federal de Santa Catarina (UFSC). Professor do Programa de Pós-Graduação em Tecnologia e Sociedade da Universidade Tecnológica Federal do Paraná (UTFPR). Slabiakjr@gmail.com

${ }^{8}$ Doutor em Engenharia de Produção pela Universidade Federal de Santa Catarina (UFSC). Professor do Programa de Pós-Graduação em Tecnologia e Sociedade da Universidade Tecnológica Federal do Paraná (UTFPR).christianlsilva76@gmail.com
} 
As áreas naturais em ambientes urbanos têm uma grande importância para as cidades. Elas contribuem com a manutenção de ecossistemas, servem de abrigo e refúgio para espécies da fauna e da flora, funcionam como parte da infraestrutura de uma região, contribuem para a regulação climática e sequestro de carbono, além de gerar inúmeros outros benefícios sociais e econômicos (SERRET et al., 2014; DEMUZERE et al., 2014). As cidades, porém, têm cada vez menos espaços verdes e geram pressão cada vez maior sobre os ecossistemas, pondo em risco também estes benefícios (DENNIS; JAMES, 2016). Por esta razão a conservação de áreas verdes remanescentes é cada vez mais importante.

O conceito de serviços ecossistêmicos descreve os benefícios aos humanos que são provenientes de ecossistemas (MILLENNIUM..., 2005). Parte destes serviços é fornecida pelos ecossistemas urbanos, que têm seu suporte em habitats em áreas verdes urbanas como jardins e parques (ELMQVIST; ALFSEN; COLDING, 2008). Os serviços ecossistêmicos são providos tanto por áreas naturais de propriedade pública, assim como as áreas particulares - todas elas contribuindo para os objetivos de desenvolvimento sustentável (SERRET et al., 2014; PANASOLO, 2015; CERRA, 2017). Nas cidades a propriedade da terra é diversa, de modo que a responsabilidade pela preservação dos ecossistemas deve ser compartiIhada entre o poder público e proprietários de terra (ELMQVIST; ALFSEN; COLDING, 2008).

O município de Curitiba, por meio de legislação própria, criou uma categoria específica de Unidade de Conservação (UC) privada, a Reserva Particular do Patrimônio Natural Municipal (RPPNM), com o objetivo de conservar e ampliar as áreas com diversidade biológica nas propriedades particulares urbanas cobertas por vegetação nativa em bom estado de conservação (PREFEITURA..., 2015). Esta categoria está em conformidade com o artigo 21 da Lei Federal no 9.985, o Sistema Nacional de Unidades de Conservação (Snuc), que estabelece a Reserva Particular do Patrimônio Natural como uma área privada, permanentemente protegida, com o objetivo de conservar a diversidade biológica (BRASIL, 2000). As reservas criadas pela legislação de Curitiba complementam outras Unidades de Conservação municipais já instituídas, que possibilitam conectividade entre as diferentes UCs e podem formar corredores ecológicos. A criação de uma RPPNM parte da iniciativa de proprietários cujo imóvel tenha ao menos 70\% de cobertura de vegetação florestal relevante ou atingido por Área de Preservação Permanente (APP) em área igual ou superior a $80 \%$ (PREFEITURA..., 2015).

Com base neste contexto, o presente trabalho tem como objetivo verificar na produção do conhecimento científico o reconhecimento dos benefícios de áreas naturais particulares em ambientes urbanos. Os estudos que subsidiam a elaboração deste artigo foram levantados por meio de uma revisão bibliográfica e permitem, ainda, a proposição de algumas recomendações para a ampliação de ambientes naturais protegidos no município de Curitiba e em outros que queiram estabelecer o mesmo tipo de política.

Este estudo foi iniciado pelo grupo de estudo Tecnologia e Meio Ambiente (Tema), o qual conduz reflexões e produção de saberes sobre conflitos e as contradições entre a tecnologia e a biosfera, intermediadas pelas ideias atuais de sustentabilidade e desenvolvimento. $\mathrm{O}$ grupo é integrante das atividades extracurriculares do Programa de Pós-Graduação em Tecnologia e Sociedade (PPGTE) da Universidade Tecnológica Federal do Paraná (UTFPR). Este Programa procura ampliar a interlocução com a comunidade externa por meio de discussões que permitam aumentar o diálogo entre a sociedade e 
a natureza em meio às forças de mudanças e a criação de modos de vida que valorizem o ser humano fundamentados nos Estudos Ciência, Tecnologia e Sociedade (PROGRAMA..., 2017).

\section{METODOLOGIA}

O desenvolvimento deste artigo deu-se a partir de uma pesquisa bibliométrica sistemática (GIL, 2010), baseada em dados secundários levantados a partir de uma revisão bibliográfica, com base em um procedimento de levantamento bibliométrico em que se pesquisou artigos sobre áreas naturais em ambientes urbanos, publicados entre os anos de 2011 e 2016, para ter um panorama atual sobre este assunto.

Os artigos foram pesquisados nas bases de dados Portal de Periódicos da Capes, Google Acadêmico, Web of Science, Scopus, Science Direct e Scielo em novembro de 2017. Foram usadas as palavras-chave em Português: áreas naturais, área verde, urbano e particular. Em Inglês usou-se "natural area", "green area", "urban" e "private". Adicionalmente, nas mesmas bases, também foram pesquisados artigos com o termo "RPPNM", em Português e Inglês, em razão de este instrumento ser único no Brasil e, seguramente, quando aparece na literatura científica trata-se de algum tipo de estudo que aborda sobre áreas naturais em ambientes urbanos.

Utilizando-se das técnicas metodológicas de pesquisa sistemática bibliométrica (ENSSLIN; ENSSLIN; PINTO, 2013), foram atribuídos os requisitos citados anteriormente, que geraram como primeiros resultados a soma de 5.831 publicações - abrangendo artigos, teses e dissertações, apresentações em eventos, entre outras - sobre as quais foram aplicados filtros iniciais (Quadro 1) para descartar as publicações que não tinham conexão direta com o tema de pesquisa deste artigo. Desse modo, como resultado final deste levantamento foram encontrados 34 artigos científicos, duas dissertações de Mestrado e uma tese de Doutorado, compatíveis com os requisitos estabelecidos para os fins desta pesquisa.

Quadro 1 - Resultados de buscas em bases de dados para composição de leitura para revisão bibliográfica por seis temas emergentes

\begin{tabular}{|c|c|c|c|c|}
\hline Base & $\begin{array}{l}\text { Quantidade } \\
\text { inicial }\end{array}$ & Filtro aplicado & $\begin{array}{c}\text { Resultado } \\
\text { final }\end{array}$ & $\begin{array}{c}\% \text { de } \\
\text { aproveitamento }\end{array}$ \\
\hline \multicolumn{5}{|c|}{ Etapa 1: Buscas } \\
\hline Capes - inglês & 28 & $\begin{array}{l}\text { Seleção de artigos } \\
\text { revisados por pares }\end{array}$ & 24 & $85,71 \%$ \\
\hline $\begin{array}{c}\text { Capes - } \\
\text { português }\end{array}$ & 27 & $\begin{array}{l}\text { Seleção de artigos } \\
\text { revisados por pares }\end{array}$ & 16 & $59,26 \%$ \\
\hline Web of Science & 18 & Sem aplicação de filtro & 18 & $100,00 \%$ \\
\hline Scopus & 33 & Sem aplicação de filtro & 33 & $100,00 \%$ \\
\hline Science Direct & 5.636 & $\begin{array}{c}\text { Priorização de artigos } \\
\text { mais relevantes e seleção } \\
\text { de tema "environmental } \\
\text { science" }\end{array}$ & 100 & $1,77 \%$ \\
\hline $\begin{array}{c}\text { Google } \\
\text { Acadêmico }\end{array}$ & 89 & Sem aplicação de filtros & 89 & $100,00 \%$ \\
\hline Total & 5.831 & & 280 & $4,80 \%$ \\
\hline \multicolumn{5}{|c|}{ Etapa 2: Leitura dos títulos } \\
\hline Portfólio & 280 & $\begin{array}{c}\text { análise dos títulos, } \\
\text { palavras-chave e resumos }\end{array}$ & 37 & $13,21 \%$ \\
\hline
\end{tabular}

Fonte: Autoria própria. 
O conteúdo encontrado na pesquisa de revisão sistemática bibliométrica (ENSSLIN; ENSSLIN; PINTO, 2013) permitiu subsidiar parcialmente a elaboração deste artigo. Para este estudo foram analisados os artigos da pesquisa de revisão bibliográfica, alinhados com seis temas (PULIGHE; FAVA; LUPIA, 2016) que precisam ser considerados pelos planejadores e tomadores de decisão quanto aos benefícios das áreas verdes ao ambiente urbano:

1) Segundo suas funções ecossistêmicas, ou seja, o funcionamento do próprio ecossistema por si e que pode gerar uma contribuição para disponibilização de serviços ecossistêmicos;

2) Capacidade de resiliência, isto é, aptidão de se recuperar e adaptar-se a anomalias ambientais, por exemplo;

3) Abordagem quanto à sustentabilidade, ou seja, as condições de durabilidade no longo prazo nas suas dimensões social, econômica e ecológica;

4) Biodiversidade - diversidade de genes, espécies e ecossistemas - e sua importância para as áreas naturais e para as cidades;

5) Nos impactos positivos de áreas verdes na saúde humana e

6) Para o bem-estar humano.

Com base na categorização apresentada, cada um dos 37 trabalhos científicos foi analisado quanto a estes seis aspectos propostos por Pulighe, Fava e Lupia (2016), o quanto e em qual contexto cada palavra-chave, ou termos similares (por ex. wellbeing e well-being, biodiversidade e diversidade biológica, resilience e resilient) são utilizados, os quais são documentados no Quadro 2.

Além da consideração destes seis temas diferentes, foi identificado o uso pouco diferenciado dos termos "área natural" e "área verde", considerando que a lei de RPPNM de Curitiba (PREFEITURA..., 2015), que deu impulso a esta pesquisa, não distingue explicitamente como se diferem as áreas naturais e as áreas verdes. Inclusive, dois dos estudos desta revisão trabalham com temas relacionados à "naturalidade", destacando-se que a análise referente a estes temas será retomada na discussão de resultados deste artigo.

Quadro 2 - Resumo da análise bibliográfica pelos seis temas emergentes (a versão completa deste quadro está disponível em: https://goo.gl/MQ2YUH

\begin{tabular}{|l|l|l|}
\hline Tema geral & Assuntos específicos & Autor(es) \\
\hline \multirow{5}{*}{$\begin{array}{l}\text { Funções } \\
\text { ecossistêmicas }\end{array}$} & $\begin{array}{l}\text { Importância geral de variedade de } \\
\text { funções ecossistêmicas }\end{array}$ & $\begin{array}{l}\text { (DENNIS; JAMES, 2016); } \\
\text { (GRISE et al., 2016); } \\
\text { (JIM, 2013); } \\
\text { (PANASOLO, 2015); } \\
\text { (RIBEIRO et al., 2013); } \\
\text { (SERRET et al., 2014). }\end{array}$ \\
\cline { 2 - 3 } & $\begin{array}{l}\text { Uso como indicador: Enhancement } \\
\text { of Urban Ecological Function; } \\
\text { Biotope Area Ratio; das Políticas } \\
\text { Públicas da União Europeia }\end{array}$ & $\begin{array}{l}\text { (CERRA, 2017); } \\
\text { (LAKES; KIM, 2012); } \\
\text { (MANEA et al., 2016). }\end{array}$ \\
\hline
\end{tabular}




\begin{tabular}{|c|c|c|}
\hline \multirow[t]{2}{*}{ Resiliência } & $\begin{array}{l}\text { Como argumento no planejamento } \\
\text { (de infraestrutura) urbano }\end{array}$ & $\begin{array}{l}\text { (ANDERSSON-SKÖLD et al., 2015); } \\
\text { (BADIU et al., 2016); } \\
\text { (DENNIS; JAMES, 2016); } \\
\text { (HERZOG, 2016). }\end{array}$ \\
\hline & $\begin{array}{l}\text { Contribuição à coesão e } \\
\text { habitabilidade }\end{array}$ & (PULIGHE et al., 2016) \\
\hline \multirow{5}{*}{ Sustentabilidade } & $\begin{array}{l}\text { Sustentabilidade como sinônimo de } \\
\text { "Iongo prazo" ou "ecologização" }\end{array}$ & $\begin{array}{l}\text { (ANDERSSON-SKÖLD et al., 2015); (DE } \\
\text { LA BARRERA et al., 2016); } \\
\text { (CARRUS et al., 2015); } \\
\text { (KOOKHAIE; MASNAVI, 2014); } \\
\text { (MANEA et al., 2016); } \\
\text { (MIDDLE et al., 2014); } \\
\text { (PÉREZ-CAMPUZANO et al., 2016); } \\
\text { (PANASOLO, 2015); } \\
\text { (RIBEIRO et al., 2013). }\end{array}$ \\
\hline & $\begin{array}{l}\text { Ameaças financeiras (restrições } \\
\text { orçamentárias), institucionais (falta } \\
\text { de mecanismos/políticas) e jurídicas }\end{array}$ & $\begin{array}{l}\text { (CHISHALESHALE et al., 2015); } \\
\text { (CLAESSENS et al., 2014); } \\
\text { (IRARRÁZAVAL, 2012); } \\
\text { (JIM, 2013); } \\
\text { (SOUZA, 2016). }\end{array}$ \\
\hline & $\begin{array}{l}\text { Relacionado à distribuição de renda, } \\
\text { progresso tecnológico ou acesso às } \\
\text { áreas verdes }\end{array}$ & $\begin{array}{l}\text { (KREKEL et al., 2016); } \\
\text { (LUVISI; LORENZINI, 2014); } \\
\text { (MINAKI; AMORIM, 2012); } \\
\text { (SHANAHAN et al., 2014). }\end{array}$ \\
\hline & $\begin{array}{l}\text { No uso dos recursos naturais ou } \\
\text { infraestrutura verde }\end{array}$ & $\begin{array}{l}\text { (BASNIAK, 2016); } \\
\text { (HERZOG, 2013); } \\
\text { (PULIGHE et al., 2016). } \\
\end{array}$ \\
\hline & $\begin{array}{l}\text { Como indicador: espaços verdes } \\
\text { urbanos per capita, Sustainable } \\
\text { Sites Initiative (SITES), Urban Green } \\
\text { Neighborhood Green Index }\end{array}$ & $\begin{array}{l}\text { (BADIU et al., 2016); } \\
\text { (CERRA, 2017); } \\
\text { (GUPTA et al., 2012). }\end{array}$ \\
\hline \multirow{5}{*}{ Biodiversidade } & $\begin{array}{l}\text { Promoção geral da biodiversidade } \\
\text { nas cidades por áreas verdes } \\
\text { e apoio à provisão de serviços } \\
\text { ecossistêmicos }\end{array}$ & $\begin{array}{l}\text { (ANDERSSON-SKÖLD et al., 2015); } \\
\text { (CARRUS et al., 2015); } \\
\text { (JIM, 2013); } \\
\text { (LAKES; KIM, 2012); } \\
\text { (PANASOLO, 2015); } \\
\text { (PÉREZ-CAMPUZANO et al., 2016); } \\
\text { (PULIGHE et al., 2016); } \\
\text { (RIBEIRO et al., 2013); } \\
\text { (VANDERMEULEN et al., 2011). }\end{array}$ \\
\hline & $\begin{array}{l}\text { Análise do estado da biodiversidade, } \\
\text { alguns com relação a tecnologias } \\
\text { para estes fins }\end{array}$ & \begin{tabular}{|l} 
(BASNIAK, 2016); \\
(HERZOG, 2016); \\
(LUVISI; LORENZINI, 2014). \\
\end{tabular} \\
\hline & $\begin{array}{l}\text { Promoção da biodiversidade como } \\
\text { objetivo de políticas e programas }\end{array}$ & $\begin{array}{l}\text { (BASNIAK, 2016); } \\
\text { (CERRA, 2017). } \\
\end{array}$ \\
\hline & $\begin{array}{l}\text { Participação na conservação da } \\
\text { biodiversidade e seu valor social }\end{array}$ & $\begin{array}{l}\text { (DENNIS; JAMES, 2016); } \\
\text { (HAND et al., 2016); } \\
\text { (MANEA et al., 2016); } \\
\text { (MIDDLE et al., 2014); } \\
\text { (SPARTZ; SHAW, 2011). }\end{array}$ \\
\hline & $\begin{array}{l}\text { Áreas particulares (de empresas) } \\
\text { têm potencial na promoção } \\
\text { de corredores ecológicos e } \\
\text { biodiversidade }\end{array}$ & $\begin{array}{l}\text { (SERRET et al., 2014); } \\
\text { (SHANAHAN et al., 2014). }\end{array}$ \\
\hline
\end{tabular}




\begin{tabular}{|c|c|c|}
\hline \multirow{5}{*}{ Saúde humana } & $\begin{array}{l}\text { Diminuição de calor, redução de } \\
\text { emissões, saúde mental (individual } \\
\text { e pública) e redução de iniquidades } \\
\text { de renda }\end{array}$ & $\begin{array}{l}\text { (ANDERSSON-SKÖLD et al., 2015); } \\
\text { (BADIU et al., 2016); } \\
\text { (BARÓ et al., 2015); } \\
\text { (BOTTALICO et al., 2016); } \\
\text { (CLAESSENS et al., 2014). }\end{array}$ \\
\hline & Prevenção de doenças & (HERZOG, 2016). \\
\hline & $\begin{array}{l}\text { Proximidade a áreas verdes e } \\
\text { benéfico à saúde }\end{array}$ & $\begin{array}{l}\text { (BERTRAM; REHDANZ, 2015); } \\
\text { (HAND et al., 2016); } \\
\text { (KREKEL et al., 2016); } \\
\text { (MANEA et al., 2016); } \\
\text { (ODE SANG et al., 2016); } \\
\text { (PANASOLO, 2015); } \\
\text { (PULIGHE et al., 2016); } \\
\text { (SERRET et al., 2014). }\end{array}$ \\
\hline & $\begin{array}{l}\text { Relação stress ambiental com stress } \\
\text { social }\end{array}$ & $\begin{array}{l}\text { (CARRUS et al., 2015); } \\
\text { (DENNIS; JAMES, 2016); } \\
\text { (MIDDLE et al., 2014). }\end{array}$ \\
\hline & $\begin{array}{l}\text { Relação proximidade a áreas verdes } \\
\text { com escolhas (alimentares / de } \\
\text { mobilidade) que contribuem à } \\
\text { diminuição no custo à saúde } \\
\end{array}$ & $\begin{array}{l}\text { (MIDDLE et al., 2014); } \\
\text { (VANDERMEULEN et al., 2011). }\end{array}$ \\
\hline \multirow{9}{*}{ Bem-estar humano } & $\begin{array}{l}\text { Importância de áreas verdes para a } \\
\text { manutenção da qualidade de vida } \\
\text { ou o bem-estar }\end{array}$ & $\begin{array}{l}\text { (BADIU et al., 2016); } \\
\text { (BARÓ et al., 2015); } \\
\text { (BERTRAM; REHDANZ, 2015); } \\
\text { (DENNIS; JAMES, 2016); } \\
\text { (KOOKHAIE; MASNAVI, 2014); } \\
\text { (KREKEL et al., 2016); } \\
\text { (MINAKI; AMORIM, 2012); } \\
\text { (PANASOLO, 2015); } \\
\text { (PULIGHE et al., 2016); } \\
\text { (SPARTZ; SHAW, 2011). } \\
\end{array}$ \\
\hline & $\begin{array}{l}\text { Benefícios de proximidade (vista) e } \\
\text { contato com a natureza }\end{array}$ & $\begin{array}{l}\text { (HAND et al., 2016); } \\
\text { (SHANAHAN et al., 2014). }\end{array}$ \\
\hline & $\begin{array}{l}\text { Importância de áreas públicas, } \\
\text { benefícios de áreas privadas (não } \\
\text { estudadas) }\end{array}$ & $\begin{array}{l}\text { (DE LA BARRERA et al., 2016)rd }><\text { ) } \\
\text { Cite }></ \text { EndNote }>\end{array}$ \\
\hline & Coesão e capital social & $\begin{array}{l}\text { (CLAESSENS et al., 2014); } \\
\text { (PÉREZ-CAMPUZANO et al., 2016). }\end{array}$ \\
\hline & Valores auto-relatados & (CARRUS et al., 2015); \\
\hline & $\begin{array}{l}\text { Conexão direta entre benefícios à } \\
\text { saúde e ao bem-estar }\end{array}$ & $\begin{array}{l}\text { (CLAESSENS et al., 2014); } \\
\text { (HAND et al., 2016); } \\
\text { (SERRET et al., 2014). }\end{array}$ \\
\hline & Uso como indicador ou variável & (LAKES; KIM, 2012) \\
\hline & Diferenças de gênero & (ODE SANG et al., 2016) \\
\hline & $\begin{array}{l}\text { Grau de "naturalidade" de espaços } \\
\text { verdes; redução em bem-estar por } \\
\text { áreas mau-mantidas }\end{array}$ & $\begin{array}{l}\text { (ANDERSSON-SKÖLD et al., 2015); } \\
\text { (ODE SANG et al., 2016). }\end{array}$ \\
\hline
\end{tabular}

Fonte: Autoria própria.

Os procedimentos metodológicos revelaram-se adequados para se conhecer a produção científica sobre áreas naturais em ambientes urbanos. O portfólio levantado apresentou publicação científica nacional e internacional apropriada para se conhecer as características e aspectos em torno dos ecossistemas encontrados nessas áreas das cidades. 


\section{REVISÃO SOBRE OS BENEFÍCIOS DA CONSERVAÇÃO DE ÁREAS NATURAIS EM AMBIENTES URBANOS}

As áreas naturais em ambientes urbanos e periurbanos são responsáveis por gerar benefícios tangíveis e intangíveis de alta importância para o bem-estar humano, particularmente em cidades médias e grandes (ANDERSSON-SKÖLD et al., 2015; BERTRAM; REHDANZ, 2015; KREKEL; KOLBE; WÜSTEMANN, 2016; PULIGHE; FAVA; LUPIA, 2016).

Estes benefícios derivados da natureza, isto é, serviços ecossistêmicos, são divididos em várias classes. Aqui se optou pela classificação da Avaliação Ecossistêmica do Milênio (MILLENNIUM..., 2005), que categoriza tais serviços como de suporte, regulação, provisão e culturais. Os serviços de regulação e de provisão muitas vezes são mais tangíveis, e a relação direta deles com áreas verdes fica mais evidente. Um dos desafios, no entanto, é o entendimento da complexidade dos valores apresentados, principalmente nos serviços intangíveis que não são facilmente mensuráveis, como os culturais e os que contribuem para a saúde humana e o bem-estar humano e que influenciam a qualidade de vida urbana (ODE SANG et al., 2016).

À medida que as áreas urbanas crescem, as cidades estão exigindo cada vez mais espaços para instalação de habitantes e atividades econômicas. Paisagens urbanas normalmente configuram extrema fragmentação de habitats. Espaços livres urbanos podem constituir fragmentos mais ou menos isolados numa matriz de espaços construídos. Quanto maior a fragmentação, maiores as dificuldades de dispersão de organismos, manutenção da diversidade biológica e provisão de serviços ecossistêmicos (ELMQVIST; ALFSEN; COLDING, 2008). Goddard, Dougill e Benton (2013, p. 266 apud CERRA, 2017) apontam que "coletivamente, a gestão sustentável de jardins privados tem enorme potencial para construir a resiliência ecológica nas cidades". Neste sentido, Angeoletto et al. (2015) citam o caso de Maringá (PR), em que jardins domésticos podem ter diversidade biológica tão rica quanto à de áreas de vegetação nativa, e o planejamento pode orientar para o aumento desta riqueza e, consequentemente, dos benefícios para a fauna e os cidadãos.

Levando essa perspectiva para os trabalhos analisados nesta pesquisa de levantamento bibliográfico, funções ecossistêmicas são consideradas, de uma forma ou outra, em 9 dos 37 trabalhos científicos analisados, observando-se que Jim (2013) aponta que a variedade de funções ecossistêmicas presentes em uma área urbana precisa ser analisada de forma holística para que sejam incorporadas no planejamento de áreas verdes urbanas e infraestrutura verde. Todos os autores que incluem funções ecossistêmicas nas suas perspectivas confirmam direta ou indiretamente que elas são importantes, mas que em si são pouco consideradas no planejamento e nas pesquisas, como informam, por exemplo Serret et al. (2014).

A resiliência das cidades é um tema mais específico que é tratado somente por cinco dos autores, ainda que todos concordem que as áreas e a infraestrutura verde são consideradas como chave (HERZOG, 2016) e que a resiliência é um argumento forte em favor das áreas verdes urbanas (DENNIS; JAMES, 2016), no entanto esse fator não é aprofundado nos estudos analisados. 
A contribuição das áreas verdes urbanas para o desenvolvimento sustentável é relatada por 23 dos 37 trabalhos. Este tema complexo é tratado de formas muito variadas, por exemplo por Gupta et al. (2012), que aplicaram o Urban Neighborhood Green Index (Ungi) na cidade de Delhi, na Índia, ou por Panasolo (2015), que considera o caso do potencial construtivo em Curitiba como medida de desenvolvimento sustentável. O uso do índice Ungi é proposto como forma de ter uma ferramenta específica de apoio à decisão para alinhar os processos de planejamento com os princípios da sustentabilidade. No caso curitibano, o autor propõe "uma reestruturação do Programa das Reservas Particulares do Patrimônio Natural Municipal para garantir a preservação, conservação e uso sustentável dos recursos" (PANASOLO, 2015, p. 18). Autores como Minaki e Amorim (2012), Jim (2013) e Luvisi e Lorenzini (2014) acrescentam o papel importante do uso de tecnologias apropriadas para atingir uma forma de desenvolvimento sustentável (PANASOLO, 2015, p. 18).

O tema da biodiversidade é tratado em 20 dos trabalhos científicos analisados. Cerra (2017) c o exemplo da prefeitura de Curitiba, com sua iniciativa de RPPNM, como instituição que administra programas complementares de conservação de habitats e de planejamento espacial e paisagístico. Trabalha com casos de ecologia cívica ${ }^{9}$ e iniciativas de instituições públicas nos Estados Unidos da América e no Japão, para acrescentar a possibilidade de cooperação institucional na governança urbana. Outros estudos discutem a conectividade ecológica ${ }^{10}$ (RIBEIRO; BORGO; MARANHO, 2013; SERRET et al., 2014; PULIGHE; FAVA; LUPIA., 2016), a importância para a manutenção do fluxo dos serviços ecossistêmicos (LAKES; KIM, 2012; HERZOG, 2016; SERRET et al., 2014; MANEA et al., 2016) e também aspectos de participação, percepção social e acesso equitativo à biodiversidade - que vai além do acesso equitativo aos espaços verdes (DENNIS; JAMES, 2016; HAND et al., 2016; CERRA, 2017). Shanahan et al. (2014) chamam a atenção para a importância de fatores de manejo em propriedades privadas que influenciam altamente a sua biodiversidade.

Saúde humana é tratado por 18 dos trabalhos aqui estudados, dos quais todos confirmam efeitos positivos de áreas verdes e inclusive ressaltam que esta conexão já está bem-estabelecida na literatura acadêmica (SERRET et al., 2014). Os efeitos positivos para a saúde humana são inúmeros. Entre os mais comuns citados na literatura estão a regulação do clima por redução de emissões e calor (CLAESSENS et al., 2014; ANDERSSON-SKÖLD et al., 2015; BARÓ et al., 2015; BOTTALICO et al., 2016) e a mitigação de estresse, assim como melhorias gerais na saúde mental e psicológica (MIDDLE et al., 2014; SHANAHAN et al., 2014; ANDERSSON-SKÖLD et al., 2015; BERTRAM; REHDANZ, 2015; DENNIS; JAMES, 2016; HAND et al., 2016; MANEA et al., 2016). Além disso, muitas pesquisas tratam juntos (quando saúde é usada como indicador) ou pelo menos estabelecem uma conexão próxima entre benefícios para saúde e bem-estar (SERRET et al., 2014; BERTRAM; REHDANZ, 2015; ODE SANG et al., 2016; PULIGHE; FAVA; LUPIA, 2016).

\footnotetext{
${ }^{9}$ Práticas ambientais de iniciativa comunitária, que promovem a intervenção em espaços livres de modo a fomentar a provisão de serviços ecossistêmicos e a promover uma melhora no ambiente (KRASNY; TIDBALL, 2015)

${ }^{10}$ É o grau em que a paisagem favorece ou impede o fluxo de energia, material, nutrientes, espécies, pessoas, e está diretamente relacionada à manutenção dos serviços ecossistêmicos. É o oposto da fragmentação, que ocorre principalmente em paisagens urbanas (AHERN, 2007).
} 
Os benefícios de áreas verdes para o bem-estar humano são discutidos em 20 dos trabalhos levantados. Pelo caráter qualitativo muitos dos autores abordam o bem-estar por estudos de percepção. São tratados aspectos de proximidade ou acesso às áreas verdes em relação ao bem-estar (LAKES; KIM, 2012; SHANAHAN et al., 2014; DENNIS; JAMES, 2016; KREKEL; KOLBE; WÜSTEMANN, 2016; PÉREZ-CAMPUZANO; AVILA-FOUCAT; PEREVOCHTCHIKOVA, 2016); questões de educação (ambiental) facilitadas pelas áreas verdes (ANDERSSON-SKÖLD et al., 2015; HAND et al., 2016) e aspectos de recreação nesses espaços (SPARTZ; SHAW, 2011; CARRUS et al., 2015).

Ao serem somados a fatores sociodemográficos, as percepções dos valores mudam significativamente. Isto é sustentado em estudos em diferentes lugares como Berlim, Cidade do México, Boston, Bucareste e Seul (BERTRAM; REHDANZ, 2015; PÉREZ-CAMPUZANO; AVILA-FOUCAT; PEREVOCHTCHIKOVAe, 2016; PULIGHE; FAVA; LUPIA, 2016). Desse modo, é importante a inclusão dos stakeholders locais no processo de avaliação e mapeamento (PULIGHE; FAVA; LUPIA, 2016).

Conclui-se nesta análise um equilíbrio nos números de artigos que tratam quatro das seis categorias (saúde, bem-estar, biodiversidade e desenvolvimento sustentável), variando entre 18 e 23 publicações. Por outro lado, o número de artigos que tratou das categorias funções ecossistêmicas e resiliências apresentou-se como baixo - nove e cinco, respectivamente.

\section{DISCUSSÃO}

Diante do exposto através da revisão sistemática bibliográfica percebe-se que as estratégias voluntárias para o realce ecológico urbano nas propriedades privadas fornecem oportunidades de atender algumas das necessidades em favor da manutenção do patrimônio natural e dos benefícios de seus serviços ecossistêmicos, mas percebe-se que estas estratégias carecem de maior profundidade de investigação científica.

Ainda que o portfólio de pesquisas levantadas para a construção deste artigo tenha sido delimitado com a busca de textos orientados a relacionar as áreas naturais de propriedade privada, é interessante notar que muitos destes estudos somente consideram áreas públicas como provedoras dos benefícios da conservação da natureza para a sociedade. Ainda assim, há os que reconhecem a importância de áreas particulares, mas não focam os estudos sobre elas (DE LA BARRERA; REYES-PAECKE; BANZHAF., 2016; CERRA, 2017) .

Destacam-se também as pesquisas de Cerra (2017) e de Serret et al. (2014) que apontam o possível "aprimoramento da função ecológica urbana" (SALMON-SAFE, 2014, apud. SERRET et al., 2014) por áreas particulares e de empresas, caso estas fossem integradas em estratégias de gerenciamento ambiental pelos órgãos públicos.

A atenção das RPPNM curitibanas como foco de estudo de Cerra (2017) e de Panasolo (2015) dão especificidade e forte correspondência para se entender os benefícios gerados pelas áreas naturais de propriedade particular existentes nas cidades. Esta constatação, aliás, tem equivalência com os objetivos da iniciativa governamental da prefeitura de Curitiba ao criar uma legislação a respeito das reservas. A legislação de RPPNM em Curitiba (PREFEITURA..., 2015) é aplicável a áreas atingidas por Bosques Na- 
tivos Relevantes ou Área de Preservação Permanente (APP), de modo que esta pesquisa concentrou-se na busca por benefícios de áreas naturais, com o sentido de áreas cobertas por vegetação nativa.

$\mathrm{Na}$ literatura analisada também foi encontrado o uso da categoria de áreas ou espaços verdes, em que alguns textos diferenciam os serviços ecossistêmicos providos por áreas verdes de vegetação mais densa, daqueles de vegetação menos densa; e alguns autores em função do nível de biodiversidade (CARRUS et al., 2015), no entanto nenhum diferencia claramente os bosques nativos.

A ausência da discussão em torno das áreas privadas na maioria dos artigos sugere que ainda é incomum, em âmbito global, a existência de instrumentos governamentais que incentivem a criação de reservas particulares e que orientem a preservação dessas áreas. Os temas discutidos nesta seção concentram-se, portanto, na pluralidade dos benefícios das áreas naturais em ambientes urbanos, mas, ao mesmo tempo, constata-se uma carência de pesquisas sobre estes espaços em comparação às áreas públicas. Assim, encaminha-se para uma concordância sobre a necessidade de ampliar o campo de conhecimento sobre este assunto.

\section{CONSIDERAÇÕES FINAIS}

Esta pesquisa permite constatar que há na literatura acadêmica o reconhecimento da importância das áreas naturais urbanas, principalmente quanto aos aspectos de desenvolvimento sustentável, biodiversidade, saúde e bem-estar. Essas áreas são suporte para uma série de serviços ecossistêmicos fundamentais para o equilíbrio e a manutenção das condições de vida na cidade. Observa-se que as áreas públicas e as particulares possuem um papel importante em prover benefícios para toda a comunidade.

Neste artigo foi possível cumprir com o objetivo central de apresentar uma revisão bibliográfica sobre a importância das áreas naturais em ambientes urbanos, principalmente a relevância de áreas particulares, buscando no referencial teórico uma base científica que potencialize a estruturação de políticas públicas e boas práticas aplicadas às RPPNMS.

Como proposta de trabalho futuro destaca-se a elaboração de índices e indicadores a serem utilizados na estruturação de políticas públicas referentes ao tema, como é sugerido por Gupta et al. (2012).

Além disso, instrumentos públicos para considerar as reservas particulares como áreas oficialmente protegidas (com reconhecimento legal e governamental) poderiam favorecer o papel do domínio privado em ações de proteção do patrimônio natural que geram benefícios para toda a coletividade. Nesse sentido, o mecanismo curitibano de RPPNM indica acerto tanto para envolver proprietários de áreas na conservação da natureza como estimular a atenção de pesquisadores sobre tal envolvimento e seus resultados para o meio ambiente e toda a sociedade.

Ao atestar essas percepções, recomenda-se estudos sobre a mobilização e adesão de particulares na criação de RPPNMs em Curitiba e os benefícios particulares de sua existência para a coletividade. Também recomenda-se a comparação entre a quantidade de áreas naturais públicas e particulares, nas perspectivas existentes e potenciais, para se discutir alcance e necessidade de envolvimento de privados nos esforços de manutenção do patrimônio natural. 


\section{REFERÊNCIAS}

AHERN, J. Green infrastructure for cities: the spatial dimension. In: NOVOTNY, Vladimir; BROWN, Paul (ed.). Cities of the Future: Towards Integrated Sustainable Water and Landscape Management. London: IWA Publishing, 2007. p. 267-283.

ANDERSSON-SKÖLD, Yvonne et al. An integrated method for assessing climate-related risks and adaptation alternatives in urban areas. Climate Risk Management, v. 7, p. 31-50, 2015. Disponível em: https://doi. org/10.1016/j.crm.2015.01.003. Acesso em: 21 mar. 2017.

ANGEOLETTO, F. et al. Ecología urbana: La ciencia interdisciplinaria del planeta ciudad. Desenvolvimento em Questão, v. 13, n. 32, p. 6-20, 2015.

ASSOCIAÇÃO DOS PROTETORES DE ÁREAS VERDES EM CURITIBA E REGIÃO METROPOLITANA. Curitiba. RPPNMs. Oficializadas em Curitiba, Curitiba, 2016. Disponível em: http://apavecuritiba.blogspot.com.

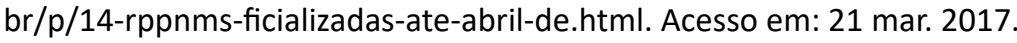

BARÓ, Francesc et al. Mismatches between ecosystem services supply and demand in urban areas: A quantitative assessment in five European cities. Ecological Indicators, v. 55, p. 146-158, 2015. Disponível em: https://doi.org/10.1016/j.ecolind.2015.03.013. Acesso em: 20 mar. 2017.

BERTRAM, Christine; REHDANZ, Katrin. The role of urban green space for human well-being. Ecological Economics, v. 120, p. 139-152, 2015. Disponível em: https://doi.org/10.1016/j.ecolecon.2015.10.013. Acesso em: 20 mar. 2017.

BOTTALICO, Francesca et al. Air Pollution Removal by Green Infrastructures and Urban Forests in the City of Florence. Agriculture and Agricultural Science Procedia, v. 8, p. 243-251, 2016. Disponível em: https:// doi.org/10.1016/j.aaspro.2016.02.099. Acesso em: 20 mar. 2017.

BRASIL. Lei no 9.985 de 18 de julho de 2000. Sistema Nacional de Unidades de Conservação. Brasília: Presidência da República; Casa Civil; Subchefia para Assuntos Jurídicos, 18 jul. 2000.

CARRUS, Giuseppe et al. Go greener, feel better? The positive effects of biodiversity on the well-being of individuals visiting urban and peri-urban green areas. Landscape and Urban Planning, v. 134, p. 221-228, 2015. Disponível em: https://doi.org/10.1016/j.landurbplan.2014.10.022. Acesso em: 13 dez. 2016.

CERRA, J. F. Emerging strategies for voluntary urban ecological stewardship on private property. Landscape and Urban Planning, v. 157, p. 586-597, 2017. Disponível em: 10.1016/j.landurbplan.2016.06.016. Acesso em: 20 mar. 2017.

CLAESSENS, J. et al. The soil-water system as basis for a climate proof and healthy urban environment: Opportunities identified in a Dutch case-study. Science of the Total Environment, v. 485-486, n. 1, p. 776784, 2014. Disponível em: https://doi.org/10.1016/j.scitotenv.2014.02.120. Acesso em: 20 mar. 2017.

CURITIBA. Lei no 14.587, de 14 de janeiro de 2015. Reestrutura o Programa das Reservas Particulares do Patrimônio Natural Municipal - RPPNM no Município de Curitiba, Revoga as Leis no 12.080, de 19 de Dezembro de 2006 e Lei no 13.899, de 9 de Dezembro de 2011. Lei Municipal de Curitiba, Curitiba, PR, 20 jan. 2015. Disponível em: https://leismunicipais.com.br/a/pr/c/curitiba/lei-ordinaria/2015/1458/14587/ lei-ordinaria-n-14587-2015-reestrutura-o-programa-das-reservas-particulares-do-patrimonio-naturalmunicipal-rppnm-no-municipio-de-curitiba-revoga-as-leis-n-12080-de-19-de-dezembro-de-2006-e-lei-n13-899-de-9-de-dezembro-de-2011. Acesso em: 18 mar. 2017.

DE LA BARRERA, Francisco; REYES-PAECKE, Sonia; BANZHAF, Ellen. Indicators for green spaces in contrasting urban settings. Ecological Indicators, v. 62, p. 212-219, 2016. Disponível em: https://doi. org/10.1016/j.ecolind.2015.10.027. Acesso em: 18 mar. 2017.

DEMUZERE, M. et al. Mitigating and adapting to climate change: Multi-functional and multi-scale assessment of green urban infrastructure. Journal of environmental management, v. 146, p. 107-115, 2014. Disponível em: https://doi.org/10.1016/j.jenvman.2014.07.025.

DENNIS, M.; JAMES, P. User participation in urban green commons: Exploring the links between access, voluntarism, biodiversity and well being. Urban Forestry \& Urban Greening, v. 15, p. 22-31, 2016. Disponível em: https://doi.org/10.1016/j.ufug.2015.11.009. Acesso em: 18 mar. 2017.

ELMQVIST, T.; ALFSEN, C.; COLDING, J. Urban Systems. In: Encyclopedia of Ecology. 2008. Disponível em: http://dx.doi.org/10.1016/B978-008045405-4.00364-5. Acesso em: 17 mar. 2017.

ENSSLIN, Leonardo; ENSSLIN, Sandra Rolim; PINTO, Hugo de Moraes. Processo de investigação e análise bibliométrica: avaliação da qualidade dos serviços bancários. Revista de Administração Contemporânea, v. 17, p. 325-349, 2013. Disponível em: http://dx.doi.org/10.1590/S1415-65552013000300005. Acesso em: 11 jun. 2017. GIL, Antonio Carlos. Como elaborar projetos de pesquisa. 5. ed. São Paulo: Atlas, 2010.

GUPTA, Kshama et al. Urban Neighborhood Green Index - A measure of green spaces in urban areas. Landscape and Urban Planning, v. 105, n. 3, p. 325-335, 2012. Disponível em: https://doi.org/10.1016/j. landurbplan.2012.01.003. Acesso em: 17 mar. 2017.

HAND, K. L. et al. A novel method for fine-scale biodiversity assessment and prediction across diverse urban landscapes reveals social deprivation-related inequalities in private, not public spaces. Landscape and Urban Planning, v. 151, p. 33-44, 2016. Disponível em: https://doi.org/10.1016/j. landurbplan.2016.03.002. Acesso em: 17 mar. 2017. 
HERZOG, Cecilia. A multifunctional green infrastructure design to protect and improve native biodiversity in Rio de Janeiro. Landscape and Ecological Engineering, v. 12, n. 1, p. 141-150, 2016. Disponível em: https://doi.org/10.1007/s11355-013-0233-8. Acesso em: 17 mar. 2017.

JIM, C. Y. Sustainable urban greening strategies for compact cities in developing and developed economies. Urban Ecosystems, v. 16, n. 4, p. 741-761, 2013. Disponível em: https://doi.org/10.1007/s11252-0120268-x. Acesso em: 10 mar. 2017.

KRASNY, M. E.; TIDBALL, K. G. Adaptation and transformation from the ground up. Civic ecology. Cambridge: MIT Press, 2015. Disponível em: https://doi.org/10.7551/mitpress/9780262028653.001.0001 KREKEL, Christian; KOLBE, Jens; WÜSTEMANN, Henry. The greener, the happier? The effect of urban land use on residential well-being. Ecological Economics, v. 121, p. 117-127, 2016. Disponível em: https://doi. org/10.1016/j.ecolecon.2015.11.005. Acesso em: 11 mar. 2017.

LAKES, Tobia; KIM, Hyun-Ok. The urban environmental indicator "Biotope Area Ratio". An enhanced approach to assess and manage the urban ecosystem services using high resolution remotesensing. Ecological Indicators, v. 13, n. 1, p. 93-103, 2012. Disponível em: https://doi.org/10.1016/j. ecolind.2011.05.016. Acesso em: 11 mar. 2017.

LUVISI, Andrea; LORENZINI, Giacomo. RFID-plants in the smart city: Applications and outlook for urban green management. Urban Forestry \& Urban Greening, v. 13, n. 4, p. 630-637, 2014. Disponível em: https://doi.org/10.1016/j.ufug.2014.07.003. Acesso em: 16 mar. 2017

MANEA, Gabriela et al. Arguments for Integrative Management of Protected Areas in the Cities - Case Study in Bucharest City. Procedia Environmental Sciences, v. 32, p. 80-96, 2016. Disponível em: https:// doi.org/10.1016/j.proenv.2016.03.014. Acesso em: 16 mar. 2017.

MIDDLE, Isaac et al. Integrating community gardens into public parks: An innovative approach for providing ecosystem services in urban areas. Urban Forestry \& Urban Greening, v. 13, n. 4, p. 638-645, 2014. Disponível em: https://doi.org/10.1016/j.ufug.2014.09.001. Acesso em: 11 mar. 2017.

MILLENNIUM ECOSYSTEM ASSESSMENT. Millennium Ecosystem Assessment. Ecosystems and Human Well-being: Synthesis. Washington, DC: Island Press, 2005.

MINAKI, C; AMORIM, M. O espaço geográfico em análises. A qualidade ambiental urbana na legislação municipal: exemplo do plano diretor de Araçatuba-SP. Revista da UFPR RA'EGA, v. 25, p. 218-251, 2012. Disponível em: http://revistas.ufpr.br/raega/article/view/28012/18646. Acesso em: 16 mar. 2017.

ODE SANG, Åsa et al. The effects of naturalness, gender, and age on how urban green space is perceived and used. Urban Forestry \& Urban Greening, v. 18, p. 268-276, 2016. Disponível em: https://doi. org/10.1016/j.ufug.2016.06.008. Acesso em: 3 fev. 2017.

PANASOLO, Alessandro. Conservação de áreas verdes urbanas privadas: proposta de modelo de transferência do potencial construtivo como estímulo para a criação de Reservas Particulares do Patrimônio Natural - RPPNMs em Curitiba. 2015. 162 f. f. Dissertação (Mestrado) - Programa de PósGraduação em Engenharia Florestal, Universidade Federal do Paraná, Curitiba, 2015.

PÉREZ-CAMPUZANO, Enrique; AVILA-FOUCAT, V. S.; PEREVOCHTCHIKOVA, María. Environmental policies in the peri-urban area of Mexico City: The perceived effects of three environmental programs. Cities, v. 50, p. 129-136, 2016. Disponível em: https://doi.org/10.1016/j.cities.2015.08.013.

PREFEITURA MUNICIPAL DE CURITIBA. Reestrutura: O Programa das Reservas Particulares do Patrimônio Natural Municipal - RPPNM no Município de Curitiba. 2015. Disponível em: https://leismunicipais.com. br/a/pr/c/curitiba/lei-ordinaria/2015/1458/14587/lei-ordinaria-n-14587-2015-reestrutura-o-programadas-reservas-particulares-do-patrimonio-natural-municipal-rppnm-no-municipio-de-curitiba-revoga-asleis-n-12080-de-19-de-dezembro-de-2006-e-lei-n-13-899-de-9-de-dezembro-de-2011.

PROGRAMA DE PÓS-GRADUAÇÃO EM TECNOLOGIA E SOCIEDADE. Sobre. 2017. Disponível em: http:// portal.utfpr.edu.br/cursos/coordenacoes/stricto-sensu/ppgte/sobre

PULIGHE, Giuseppe; FAVA, Francesco; LUPIA, Flavio. Insights and opportunities from mapping ecosystem services of urban green spaces and potentials in planning. Ecosystem Services, v. 22, Part A, p. 1-10, 2016. Disponível em: https://doi.org/10.1016/j.ecoser.2016.09.004.

RIBEIRO, Juliana Baladelli; BORGO, Marília; MARANHO, Leila Teresinha. Áreas protegidas de Curitiba (PR, Brasil) como sumidouros de $\mathrm{CO}_{2}$. Floresta, v. 43, n. 2, p. 181-190, 2013.

SERRET, Hortense et al. Potential contributions of green spaces at business sites to the ecological network in an urban agglomeration: The case of the Ile-de-France region, France. Landscape and Urban Planning, v. 131, p. 27-35, 2014. Disponível em: https://doi.org/10.1016/j.landurbplan.2014.07.003.

SHANAHAN, D. F. et al. Socio-economic inequalities in access to nature on public and private lands: A case study from Brisbane, Australia. Landscape and Urban Planning, v. 130, p. 14-23, 2014. Disponível em: https://doi.org/10.1016/j.landurbplan.2014.06.005.

SPARTZ, James T.; SHAW, Bret R. Place meanings surrounding an urban natural area: A qualitative inquiry. Journal of Environmental Psychology, v. 31, n. 4, p. 344-352, 2011. Disponível em: https://doi. org/10.1016/j.jenvp.2011.04.002. 